\title{
Reverse Innovation: Could Learning From LMIC Child Health Programmes Support HIC to Equitably and Efficiently Address Child Health Needs Post COVID-19?
}

Monica Lakhanpaul1,2*, Diana Margot Rosenthal 1,2, Maya Lakhanpaul ${ }^{3}$, Jennifer Martin ${ }^{1,4}$, Michelle Heys $^{1,5}$ and Priti Parikh ${ }^{6}$

${ }^{1}$ Population, Policy and Practice Research and Teaching Department, UCL Great Ormond Street Institute of Child Health, London, UK

${ }^{2}$ UCL Collaborative Centre for Inclusion Health, UK

${ }^{3}$ University of Manchester, UK

${ }^{4}$ Women in Global Health Finland

${ }^{5}$ Specialist Children and Young People's Services, East London NHS Foundation Trust, UK

${ }^{6} U C L$ Engineering for International Development Centre, UK

It has been recognised that the first 1000 days (now extended to age 5) of a child's life are fundamental for growth and brain development [1]. Furthermore, it is considered a critical time across the life course when early intervention can mitigate long-term consequences of malnutrition, infections, and other recognised public health problems leading to conditions such as developmental delay, obesity, diabetes and asthma later in life [2]. The unprecedented global crisis, through the corona virus disease 2019 (COVID-19), will undoubtedly have an impact on child health for many years to come in low and low-middle income countries (LIC/LMIC) but also high-income countries (HIC). The disease has led to a number of barriers hindering the implementation of child health programmes in $\mathrm{HIC}$, which will further contribute to health inequalities especially for those already most marginalized in society. Public health issues such as the decline in children being vaccinated, as observed in England, are likely to become more evident post COVID-19 [3]. There is an urgency to consider how we need to modify our current public health strategies in HIC to mitigate for these secondary negative consequences. Looking towards and learning from conflict settings and areas of humanitarian crisis in LIC/LMIC may be a place to start. The process, whereby HIC adopt interventions developed in LIC/LMIC is most frequently described as Reverse Innovation [4].

In HIC, as with many LIC/LMIC, barriers to the implementation of current child public health programmes include health service physical and human resource limitations, which are becoming even more restricted during the COVID-19 pandemic. In the UK, healthcare professionals have been redeployed from preventative activities to provide support on the frontline and non-essential appointments have been drastically reduced in order for health care systems to tackle the acute burden of COVID-19 patients, physically and financially. This has meant that healthcare professionals who would routinely carry out screening programmes for young children in the community may no longer be available. In addition, due to the high virulence of the disease and the need to reduce non-essential face-to-face interactions, it has become a challenge to implement the Healthy Child Programme [5] and other early prevention initiatives. Consequently, routine appointments which include screening programmes, such as dental appointments and hearing clinics have been put on hold [5]. Furthermore, where these programmes are still running (e.g. immunisations), parents may elect or be unable to take their children for their routine immunisations (data currently unavailable) risking a rise in communicable diseases such as measles, mumps and rubella.

In addition, many countries have introduced the measure of a 'lock down' limiting access to public spaces, healthcare and supermarkets. These restrictions reduce opportunities for physical activity and access to the outdoor environment, particularly for households that do not have a garden, in-

*Corresponding author: Prof. Monica Lakhanpaul, Faculty of Population Health Sciences, UCL Great Ormond, Street Institute of Child Health, 30 Guilford Street, London WC1N 1EH, UK

Received: June 03, 2020

Accepted: July 01, 2020

Published online: July 03, 2020

Citation: Lakhanpaul M, Rosenthal DM, Lakhanpaul M, et al. (2020) Reverse Innovation: Could Learning From LMIC Child Health Programmes Support HIC to Equitably and Efficiently Address Child Health Needs Post COVID-19?. Ann Public Health Reports 4(1):53-54 
Citation: Lakhanpaul M, Rosenthal DM, Lakhanpaul M, et al. (2020) Reverse Innovation: Could Learning From LMIC Child Health Programmes Support HIC to Equitably and Efficiently Address Child Health Needs Post COVID-19?. Ann Public Health Reports 4(1):53-54

creasing the risk of vitamin D deficiency. Iron and other nutrient deficiencies may also rise, leading to a level of malnutrition that HIC may not have seen before in this lifetime. This is especially for those living in economically-deprived circumstances where providing adequate quantity and diversity of meals maybe challenging.

We propose that HIC should learn from programmes delivered in settings such as refugee camps in LMIC. LMIC are experienced at delivering screening and early intervention programmes at scale, where there is economic restraint and low resources, a phenomenon which HIC are now experiencing. The most well-known approaches are health camps, mobile health clinics and school-based interventions, where children are screened for conditions such as malnutrition, ongoing health issues or where interventions, such as immunisation is delivered to a large number of children in a short amount of time [6-8]. The UNHCR guidance takes this a step further by promoting integration of health services, food distribution, improvement in WASH facilities and active community surveillance through existing informal networks to ensure health and well-being of residents $[8,9]$. Mobile health clinics have been utilised as a means to facilitate access to the most vulnerable and marginalised, and have been tested in HIC settings including the United States to deliver a range of services including urgent care and chronic disease management.

Our associated health and societal responses to the pandemic and the indirect impacts of COVID-19 pandemic are still being established. However, there is a real risk that many early childhood problems will become 'invisible' from society. Learning from cost-effective community-based interventions successfully implemented in resource-poor settings is essential. The time is now for HIC to re-evaluate, at least for the short term, current public health programmes by taking seriously the notion of reverse innovation and setting aside more individualised approaches in favour of one similar to those adopted in areas of humanitarian crisis. We suggest the following three public health strategies as options based on their effective implementation in LMIC: 1) Schools as venues, 2) Health camps, and 3) Mobile health clinics. These strate- gies could be a more equitable and efficient one ensuring that the current gaps in health across our society do not increase even further while also reducing the economic drain over time already implicated by the pandemic.

\section{Contributors}

$M L, M L, P P$ and $D M R$ conceived the study and drafted the manuscript. DMR, JM, MH, PP read, edited, approved and finalised the manuscript.

\section{Declaration of Interests}

We declare no competing interests.

\section{References}

1. UNICEF (2020) Early moments matter.

2. Janis Baird, Chandni Jacob, Mary Barker, et al. (2017) Developmental origins of health and disease: A lifecourse approach to the prevention of non-communicable diseases. Healthcare (Basel) 5: 14 .

3. NHS Digital. Childhood Vaccination Coverage Statistics- England 2017-18.

4. Govindarajan V, Ramamurti R (2011) Reverse innovation, emerging markets, and global strategy. Global Strategy Journal 1: 191205.

5. Department of Health and Social Care (2009) Healthy Child Programme: Pregnancy and the First 5 Years of Life.

6. International Rescue Committee (IRC) (2020) Live updates: Go inside the IRC's response to COVID-19.

7. Yu SWY, Hill C, Ricks ML, et al. (2017) The scope and impact of mobile health clinics in the United States: A literature review. Int J Equity Health 16: 178.

8. Bresee, BA Caruso, J Sales, et al. (2016) A child is also a teacher': Exploring the potential for children as change agents in the context of a school-based WASH intervention in rural Eastern Zambia. Health Educ Res 31: 521-534.

9. Refugees UNHC for Global Strategy for Public Health: A UNHCR Strategy 2014-2018 Public Health - HIV and Reproductive Health - Food Security and Nutrition - Water, Sanitation and Hygiene.

DOI: $10.36959 / 856 / 492$

Copyright: (c) 2020 Lakhanpaul M, et al. This is an open-access article distributed under the terms of the Creative Commons Attribution License, which permits unrestricted use, distribution, and reproduction in any medium, provided the original author and source are credited. 